\title{
Algenbestände als Konzentrationen organischen Materials im Meer
}

\author{
Ivka Munda und Dušan Zavodnik \\ Biološki institut Slovenske Akademije znanosti in umetnosti, Ljubljana, Jugoslavija, \\ und \\ Institut za biologiju mora Jugoslavenske Akademije znanosti i umjetnosti, Rovinj, \\ Jugoslavija
}

\begin{abstract}
Seaweeds as concentrations of organic material in the sea. Communities based on seaweeds are characterized by considerable fluctuations of several hydrographical factors, richness of plant and animal species, and great amounts of biomass. In the present paper, biomass values of algal populations are compared with those of communities from other regions in the sea. Because of the prevailing plant component, seaweed communities differ in their average chemical composition from the zoocoenoses of other regions. The densest populations of seaweeds are developed in temperate and cold seas in small depths of the mediolittoral and sublittoral zones. Since these populations show the highest mean values of biomass per surface unit, it is concluded that these concentrations of organic substance are of the greatest importance for studies concerned with the bioproductivity of the sea.
\end{abstract}

\section{EINLEITUNG}

Algenbestände unterscheiden sich in mehrerer Hinsicht von anderen Lebensgemeinschaften des Meeres. Vornehmlich machen sich hier erhebliche Variationen einiger hydrographischer Faktoren bemerkbar, auf welche wiederum in gewissem Umfang die Organismen des Phytals einwirken. Wir meinen hier vor allem die Veränderungen der Sättigungswerte gelöster Gase und der Ionenkonzentration des Meerwassers. Es ist eine Tatsache, daß der Tagesrhythmus der Variationen dieser Faktoren streng abhängig ist von den Assimilations- und Dissimilations-Prozessen lebender Organismen der Algenund Seegrasbestände (GILeT 1956, Kerneïs 1960, Ledoyer 1962, ZAvodNIK 1963); dies zeigt sich auch in der Intensität dieser Faktoren. So ist beispielsweise festgestellt worden, daß im Cystoseira barbata-Bestand bei Rovinj der Unterschied in der Sauerstoffsättigung bei Tag und Nacht sogar mehr als $30 \%$ beträgt. Demzufolge ändert sich auch der Partialdruck des Kohlendioxyds und natürlich die Alkalinität (LeDoyer 1962). Es bestehen Hinweise, daß es in den Algenbeständen weniger freie Phosphate gibt als im offenen Wasser oder oberhalb unbewachsenen Bodens, was im Hinblick auf die Stoffwechselaktivität der Pflanzen ja auch zu erwarten ist. 


\section{ERGEBNISSE UND DISKUSSION}

In Abhängigkeit von den Algenbeständen wird auch die Intensität anderer klimatischer und edaphischer Faktoren verändert. So erfolgt beispielsweise die Austrocknung der mediolitoralen Zone zur Ebbezeit viel langsamer, wenn sie mit dichten Algenbeständen bedeckt ist. Ebenso sind die Variationen der Temperatur wegen der reduzierten Evaporation kleiner. Vor allem wird der Einfluß der Wellen und der im oberen

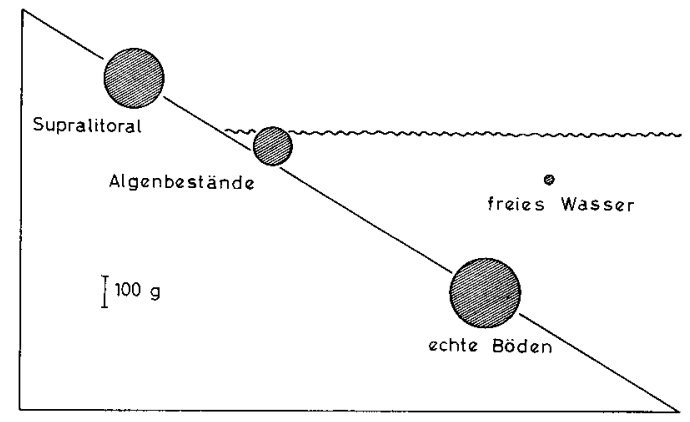

Abb. 1: Gewichtsverhältnisse der Zoobiomassen verschiedener Gebiete in Meer

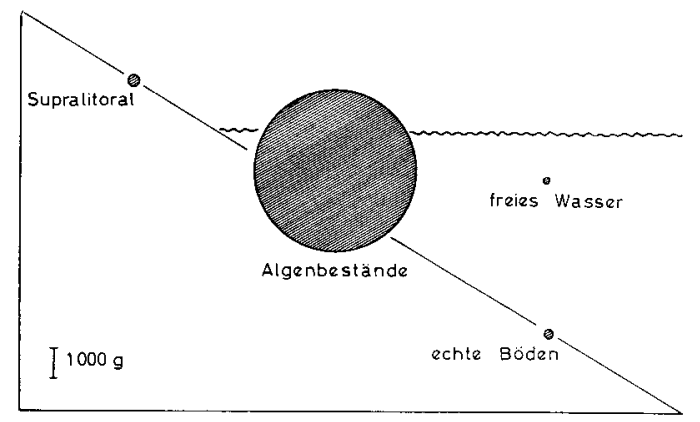

Abb. 2: Mittelwerte der Gesamtbiomassen verschiedener Gebiete im Meer

Infralitoral oft starken Flutströme gemildert. Von der Dichte und Qualität der Algenbestände ist ferner abhängig der Grad der Turbidität und der Sedimentation sowie die Beleuchtung des primären und sekundären Bodens, auf dem sich diese Bestände entwickelt haben.

Zweifellos beeinflussen all diese Faktoren die qualitative und quantitative $\mathrm{Zu}-$ sammensetzung des Bios in den Algenbeständen. Die Mannigfaltigkeit und Gliederung der Unterlage (Algen) führte zur Entwicklung der verschiedensten Tierformen, die nicht selten speziell an das Phytal adaptiert sind (haptische Formen, Färbung etc.). Trotz qualitativen Reichtums unterscheidet sich jedoch die Biomasse der Tierkomponente von Algenbeständen mit dem Mittelwert 113,9 g/m² (Abb. 1) nicht wesentlich von medio- und infralitoralen Zoocoenosen der echten Böden mit durchschnittlich 
$235,4 \mathrm{~g} / \mathrm{m}^{2}$. Die tierische Biomasse des freien Wassers ist natürlich viel geringer und übersteigt gewöhnlich nicht $10 \mathrm{~g} / \mathrm{m}^{2}$ Meeresoberfläche. Sobald man aber auch die pflanzliche Biomasse in Betracht nimmt, ändert sich das Bild vollständig (Abb. 2). Mit einem Mittelgewicht von $5565 \mathrm{~g} / \mathrm{m}^{2}$ überwiegen die Algenbestände im Mediolitoral oder im oberen Infralitoral bei weitem die Mittelwerte der Biomasse aller anderen Gebiete. Die durchschnittliche Biomasse der supralitoralen Lebensgemeinschaften der nördlichen Hemisphäre beträgt $202,5 \mathrm{~g} / \mathrm{m}^{2}$, also kaum weniger als die der infralitoralen Gemeinschaften auf echten Böden $\left(235,4 \mathrm{~g} / \mathrm{m}^{2}\right)$. Die Mittelwerte der Biomasse pelagischer Lebensgemeinschaften bleiben jedoch unter $10 \mathrm{~g} / \mathrm{m}^{2}$. Diese Werte sind natürlich nicht identisch mit dem Gewicht der organischen Materie selbst. Im Hinblick auf den hohen Wassergehalt der Organismen und auf die häufige Bildung anorganischer Skeletteile können wir nach HaRveY (1950) voraussetzen, daß auf $100 \mathrm{~g}$ Biomasse der Gemeinschaften des Pelagials oder echten Bodens annähernd $17 \mathrm{~g}$ organische Materie kommt.

Aus dem bisher Gesagten können wir schließen, daß die pflanzliche Biomasse in den Beständen der Meeresalgen durchschnittlich 48mal größer ist als die tierische Biomasse derselben Bestände. Das ist natürlich ein Durchschnittswert, in einigen Fällen übertriff die pflanzliche Biomasse die tierische auch um mehr als das 200 fache (ZAvodNIK 1965).

Sehr interessant ist der Vergleich der Biomasse eines durchschnittlichen Algenbestandes mit Mittelwerten der Biomasse einiger spezifischen Lebensgemeinschaften aus anderen Meeresgebieten (Abb. 3). Mytiliden-Gemeinschaften mit einem Mittelgewicht von

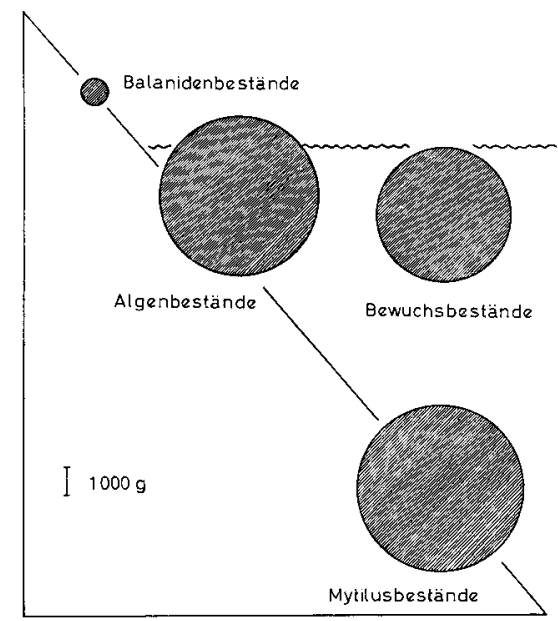

Abb. 3: Mittelwerte der Biomassen von einigen spezifischen marinen Lebensgemeinschaften

$5832 \mathrm{~g}$ auf $1 \mathrm{~m}^{2}$ Oberfläche übertreffen etwas die Algenbestände in Hinsicht auf die gesamte und besonders auf die tierische Biomasse. Nur wenig stehen hinter diesen zurück die Gemeinschaften des Bewuchses $\left(4623,7 \mathrm{~g} / \mathrm{m}^{2}\right)$ und Schwärme pelagischer Fische (etwa Sardellen), welche unseren Berechnungen nach annähernd dieselben Werte erreichen. Supralitorale Gemeinschaften mit Seepocken (Balaniden und Chthamaliden) 
mit einer durchschnittlichen Biomasse von $887,3 \mathrm{~g} / \mathrm{m}^{2}$ stehen sehr zurück. Bei Berücksichtigung der maximalen bekannten Werte derartiger Ansiedlungen ändert sich jedoch die Situation wesentlich (Abb. 4). Die dichtesten Algenbestände bilden die Braunalgen, die nach ZENKEvič (1956) in Ausnahmefällen 25 bis $28 \mathrm{~kg} / \mathrm{m}^{2}$ erreichen können. Einen der dichtesten Bestände bildet Ascopbyllum nodosum mit bis zu 10 bis $16 \mathrm{~kg} / \mathrm{m}^{2}$ in

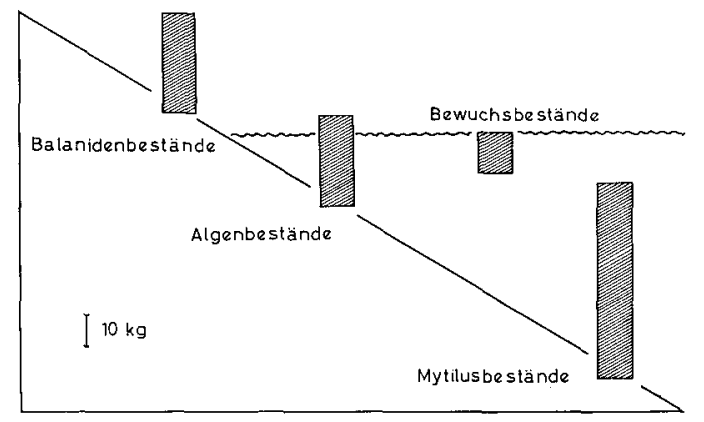

Abb. 4: Maximalwerte der Biomassen einiger spezifischer Lebensgemeinschaften

vielen Gebieten (Colman 1940, Zenkevič 1956, Munda 1964). Maximale Werte der Gemeinschaften des Bewuchses sind, soweit uns bekannt ist, im Schwarzen Meer registriert worden und betragen 8 bis über $10,6 \mathrm{~kg}$ auf $1 \mathrm{~m}^{2}$ der bewachsenen Oberfläche (Dolgopoljskaja 1954, Abadžieva-Marinov 1965). Nach Zenkevič (1956) können Bestände der Mytiliden sogar 50 bis $80 \mathrm{~kg} / \mathrm{m}^{2}$ und die der Balaniden bis zu $30 \mathrm{~kg} / \mathrm{m}^{2}$ erreichen, jedoch sind das Ausnahmewerte. Sehr dichte und gut entwickelte natürliche Bestände der Mytiliden erreichen sonst 5 bis $10 \mathrm{~kg} / \mathrm{m}^{2}$ (Mokievskij 1960, Reish 1964) und der Balaniden im Supralitoral 1 bis $2 \mathrm{~kg} / \mathrm{m}^{2}$ (Mokievskij 1960).

In den meisten Algenbeständen des Mediolitorals und oberen Infralitorals dominieren Braunalgen, vor allem Fucaceen. Die Biomasse der litoralen Braunalgen der atlantischen Küsten (Ascophyllum nodosum, Fucus vesiculosus, $F$. serratus etc.) ist bedeutend höher als im Mittelmeer. Die Fucaceen der atlantischen Küsten kommen in einer Dichte bis zu etwa $20 \mathrm{~kg} / \mathrm{m}^{2}$ vor. Die Biomasse der Algenbestände tieferer Regionen (bis etwa $20 \mathrm{~m}$ ), wo Laminaria-Arten dominieren, ist noch bedeutend größer. So entfallen zum Beispiel 18000 Tonnen Laminaria byperborea auf einen Kilometer der Küste West-Norwegens.

Im Mittelmeer kommen in den Algenbeständen vorwiegend Cystoseira und Sargassum-Arten vor, die in einer Dichte von 1 bis $9 \mathrm{~kg}$ per $\mathrm{m}^{2}$ auftreten. Die Laminariaceen des Mittelmeers sind dagegen nur durch die Art Laminaria rodriguezii vertreten und kommen nur sporadisch in einer Tiefe von 100 bis $200 \mathrm{~m}$ vor.

Es ist verständlich, daß die Verbreitung der Algenbestände von vielen Faktoren abhängt. Uns interessiert hier vor allem die Tiefe. Messungen von fast 1000 Proben haben gezeigt, daß auf der nördlichen Hemisphäre die pflanzliche Biomasse in Tiefen von 5 bis $10 \mathrm{~m}$ konzentriert ist, wo sie einen Mittelwert von $10,51 \mathrm{~kg} / \mathrm{m}^{2}$ erreicht (Abb. 5). Algenbestände erreichen sogar in der Gezeitenzone einen Mittelwert von $5,29 \mathrm{~kg} / \mathrm{m}^{2}$, in der supralitoralen Zone entwickeln sie sich jedoch selten. Ebenso schnell 
verringert sich die Biomasse der Algen in größeren Tiefen, und unter $60 \mathrm{~m}$ gibt es praktisch keine dichten Algenbestände mehr.

Im Zusammenhang mit wachsender Tiefe und plenternden Algenbeständen verändert sich natürlich auch die Proportion der lebenden Materie. Während im Mediound oberen Infralitoral die pflanzliche Materie absolut überwiegt, dominiert dem-

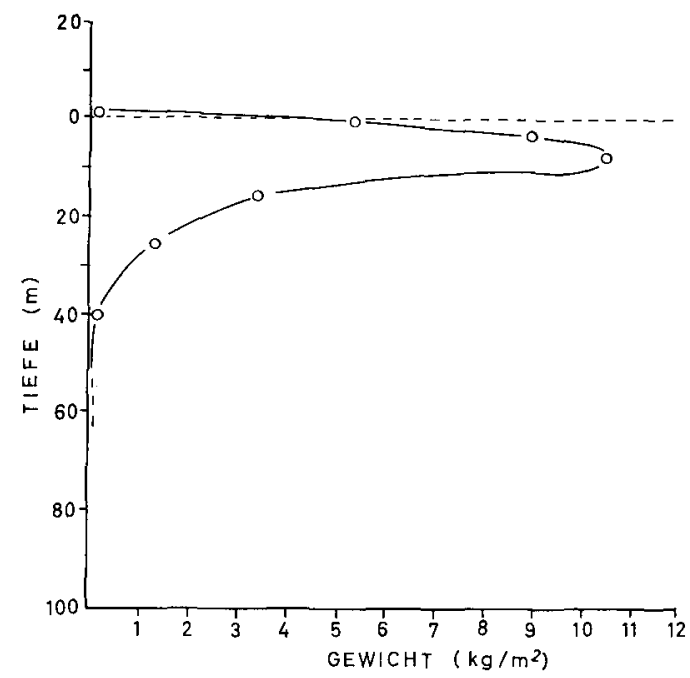

Abb. 5: Tiefenverbreitung der Algenbiomasse in den Meeren der nördlichen Hemisphäre

gegenüber im Supralitoral und auf tieferen echten Böden die tierische Materie. In dieser Hinsicht gibt es zwischen den Weltmeeren nur wenige Unterschiede. Hinsichtlich der Artenzahl überwiegen in allen Schichten und Biocoenosen die Tiere.

Die Stratifikation der organischen Materie auf dem Meeresgrund und die $\mathrm{D}$ a uerhaftigkeit der Algenbestände bedürfen noch eines besonderen Hinweises. Während sich auf festen Böden nur wenige Organismen einbohren und im Substrat leben, graben sich die Tiere in weichen Böden in den meisten Fällen ein und leben ständig im Substrat. Auf festen Böden überwiegen also Epibiosen über die Endobiosen. Da die Körper der Algen und auch des Seegrases gewöhnlich nicht am Boden selbst liegen, findet sich also die Hauptmasse der Algenbestände und Seegraswiesen über dem eigentlichen Substrat. Die Biomasse dieser Bestände ist also konzentriert in der erhöhten Schicht (strate élevée), wo sich auch die vagile Fauna am meisten aufhält. Zur Dauerhaftigkeit der Algenbestände: Die Arten, welche dichte und kompakte Bestände bilden, sind gewöhnlich mehrjährig, was besonders für Laminarien, Fucaceen und Cystoseiren gilt. Nicht selten sind die Jahreszyklen im Leben dieser Algen gut ausgeprägt; das gilt sowohl für die Pigmentation und Thalli als auch für die Veränderungen in Habitus und Dimensionen. Besonders die Cystoseiren zeigen erhebliche Variationen, so daß wir schon dem Habitus nach die einzelnen Abschnitte ihres Jahreszyklus unterscheiden können (ErCEgović 1952). Die Thalli der mehrjährigen Algen verfallen langsam, und die organische Materie ihres Körpers geht Teil um Teil langsam in 
Detritus über oder löst sich direkt im Wasser auf. Die Entfesselung der angehäuften Energie verläuft also viel langsamer als beim Phytoplankton und kann sich im Vergleich zu der für den Aufbau benötigten Zeit auch um ein bis mehrere Jahre verspäten. Wir glauben, daß gerade diesem Problem beim Studium der Produktivität der Küstenregionen die größte Aufmerksamkeit zu widmen ist.

Die chemische Zusammensetzung der Algenbestände ist wesentlich verschieden von jener der Zoocoenosen der unbewachsenen Böden; sie ist von der Artenzusammensetzung der Bestände abhängig und wuchsort- und jahreszeitbedingten Variationen unterworfen.

Die Braunalgen haben einen Aschengehalt von 15 bis 30\% ihres Trockengewichts. Ihre Trockensubstanz besteht vorwiegend aus Chloriden und ist starken jahreszeitlichen Variationen unterworfen. Der Aschengehalt der Braunalgen des Mittelmeeres scheint bedeutend höher zu sein als jener der atlantischen Arten. Die Trockensubstanz der Braunalgen beträgt im Durchschnitt 10 bis $25 \%$ des Feuchtegewichts. Den größten Teil der Algenbiomasse machen jedoch verschiedene Kohlenhydrate aus (Laminarin, Fucoidin, Alginsäure, Mannitol), während die Proteine nur einen Bruchteil von 5 bis $15 \%$ der Trockensubstanz ausmachen, die ätherlöslichen Bestandteile aber nur etwa 2 bis $6 \%$. Im Sommer und Herbst werden die meisten Kohlenhydrate synthetisiert (Mannitol, Laminarin), was üblicherweise mit einer intensiven Wachstumsperiode zusammenfällt. Die Proteine erreichen oft ein Frühlingsmaximum, während die ätherlöslichen Bestandteile konstant bleiben.

Die chemische Zusammensetzung der Algenbestände wird ebenso von den Wuchsortbedingungen beeinflußt, vor allem vom Salzgehalt. Es wurde bewiesen, daß eine erniedrigte Salinität die Synthese der Proteinstoffe beschleunigt, die Bildung des Mannitols aber zurücksetzt. Die phenolischen Komponenten der Braunalgen sind im Brackwasser niedriger als im Salzwasser. Der Gehalt der Alginsäure (20 bis 30\%), der einen wesentlichen Teil der Algenbiomasse ausmacht, ist im allgemeinen in den Mittelmeerarten niedriger als in den atlantischen Arten (Cystoseira-Arten) und auch qualitativ verschieden.

Neben den erwähnten Stoffen werden in den Algenbeständen auch zahlreiche Vitamine synthetisiert (Ascorbinsäure, Tocopherol, Niacin, Biotin, $\mathrm{B}_{12}$ etc.).

Hinsichtlich der chemischen Zusammensetzung unterscheiden sich die Tiere natürlich von den Pflanzen. Das bedeutet, daß die Konzentration der organischen Materie in den Algenbeständen ihrer Zusammensetzung nach mit der Zusammensetzung in den Zoocoenosen auf echten Böden nicht identisch ist. Ohne Berücksichtigung der Skelettteile (Korallen, Weichtiere, Bryozoen) ist die durchschnittliche Zusammensetzung der Tiere folgende: Wasser etwa $77 \%$, Fette $3 \%$, Proteine $12 \%$, Kohlenhydrate $3 \%$ und Asche etwa $5 \%$.

Im Gegensatz zu der Algenzusammensetzung überwiegen also in der tierischen Komponente Eiweißstoffe. Variationen in der prozentualen Beteiligung der einzelnen Bestandteile sind natürlich bedeutend; sie werden bedingt durch die Artzugehörigkeit der Tiere, teils auch durch die Jahreszeit beziehungsweise die Reproduktionsperiode. Leider sind die Schwankungen in der chemischen Zusammensetzung der einzelnen Tierarten noch sehr wenig bekannt; die zu Gebote stehenden Angaben beziehen sich fast ausschließlich auf die ökonomisch wichtigen Arten der Weichtiere und Fische. Es wurde 
festgestellt, daß die relativen Anteile der Eiweißstoffe, Kohlenhydrate und Asche im Laufe des Jahres nicht stark variieren, daß sich aber vor allem Fette und Wassergehalt stark verändern. So erhöht sich beispielsweise bei Muscheln und Fischen während der Reproduktionsperiode der Wassergehalt auf Kosten der Fettreserven; die Unterschiede können mehr als $7 \%$ des Gesamtgewichts des Organismus erreichen.

\section{ZUSAMMENFASSUNG}

1. Algenbestände unterscheiden sich von anderen Lebensgemeinschaften des Meeres vor allem durch erhebliche Variationen einiger hydrographischer Faktoren, durch den Reichtum der Pflanzen- und Tierarten und durch die konstanten und verhältnismäßig hohen Werte der Biomasse.

2. Mit einer Gesamtbiomasse von $5565 \mathrm{~g} / \mathrm{m}^{2}$ übertreffen die Algenbestände weit die Mittelwerte der Biomasse aller anderen Gebiete.

3. Diese hohen Werte der Biomasse werden allerdings nur durch die pflanzlichen Organismen der Algenbestände verursacht. Die tierische Biomasse weicht nicht wesentlich vom Mittelwert aller Lebensgemeinschaften ab; sie macht nur $1 / 48$ der pflanzlichen Biomasse aus. Nur wenige spezifische Lebensgemeinschaften haben gleiche oder höhere Biomassewerte (z. B. Mytiliden- und Bewuchsbestände, Fischschwärme).

4. Die Biomasse und die Qualität der Algenbestände ist vom Ort und der Wassertiefe abhängig. Algenbestände sind am dichtesten in nördlichen Meeren; die höchsten Gewichtswerte findet man in Tiefen von 5 bis $10 \mathrm{~m}$.

5. Mit zunehmender Tiefe verändert sich die Proportion zwischen pflanzlichen und tierischen Organismen; dies wiederum beeinflußt die chemische Zusammensetzung der Biocoenosen. Jahresvariationen der chemischen Zusammensetzung sind feststellbar.

6. Die Algenbestände kann man als typische Epibiosen bezeichnen.

7. Von größter Bedeutung ist die Dauerhaftigkeit der Algenbestände. Die dort organisch gebundene Energie wird erst nach mehreren Jahren frei. Bioproduktionsstudien an Algenbeständen sind daher aus ernährungswirtschaftlichen Gründen für die Gegenwart - und noch mehr für die Zukunft - von großer Wichtigkeit.

\section{ZITIERTE LITERATUR}

Arbuzova, K. S., 1963. Obrastanie $\mathrm{v}$ jugo-vostočnoj časti Baltijskogo morja. Trudy Inst. Okeanol. 70, 41-51.

BaARDSETH, E., 1954, Kvantitative tare-undersøkelser i Lofoten og Salten sommeren 1952. Rep. Norw. Inst. Seaweed Res. 6.

Bellan-SantinI, D., 1964. Etude qualitative et quantitative du peuplement à Cystoseira crinita Bory. Recl. Trav. Stn. mar. Endoume 34 (50), 249-261.

Chapman, Y. J., 1950. Seaweeds and their uses. Pitman, London, $287 \mathrm{pp}$.

Colman, J., 1940. On the faunas inhabiting intertidal seaweeds. J. mar. biol. Ass. U. K. 24, 129-183.

Dimov, I. G., 1964. Sezonni izmenenija na vidovija sestav na zooplanktona i tjaknoto vlijanie vrhu biomassata mu $\mathrm{v}$ zapadnite rajoni na Črno more. Izv. Inst. Ribov. Ribolov, Varna (Bull. Inst. Piscic. Pêche) 5, 33-44. 
DolgopoljskajA, M. A., 1954. Eksperimentaljnoe izučenie processa obrastanija v more. Trudy sevastopol biol. Sta. 8, 157-173.

Ercegović, A., 1952. Cistozire Jadrana. Fauna Flora adriat. 2, 212 pp.

Gilet, R., 1956. L'eau du milieu phytal marin. Rapp. P.-V. Réun. Commn int. Explor. scient. Mer Mediterr. 13, 195-198.

Grenager, B., 1953-58. Kvantitative undersøkelser av tareforekonster ... Rep. Norw. Inst. Seaweed Res. $3 ; 5 ; 7 ; 13 ; 21$.

Hagmeier, E., 1964. Zum Gehalt an Seston und Plankton im Indischen Ozean zwischen Australien und Indonesien. Kieler Meeresforsch. 20, 12-17.

HARver, H., 1950. On the production of living matter in the sea of Plymouth. 7. mar. biol. Ass. U. K. 29, 97-137.

Herberts, $\mathrm{CH}$, 1964. Contribution à l'étude du peuplement rocheux sessile dans la zone à Fuctes serratus L. Bull. Lab. marit. Dinard 49/50, 5-59.

KANEVA-ABADZ̆IEYA, V. \& MARINOV, T. M., 1960. Raspredelenie na zoobentosa pred blgarskoto cernomorsko krajbrežie. Trud. centraln. naucnoizsled. Inst. Ribov. Ribolov, Varna (Arb. zentr. Forschlnst. Fischzucht Fischerei) 3, 117-161.

- 1965. Morski obrastanija po blgarskoto krajbrežie na Cerno more. Izv. naǔ̌noizsled. Inst. Ribno stopanstvo Okeanogr., Varna (Proc. Res. Inst. Fish. Oceanogr.) 6, 137-144.

$\mathrm{KAx}, \mathrm{H}$, 1954. Untersuchungen zur Menge und Verteilung der organischen Substanz im Meerwasser. Kieler Meeresforsch. 10, 202-214.

Kerneïs, A., 1960. Contribution à l'étude faunistique et écologique des herbiers de Posidonies de la region de Banyuls. Vie Milien 11, 145-187.

KreY, J, 1964. Die mittlere Tiefenverteilung von Seston, Mikrobiomasse und Detritus im nördlichen Nordatlantik. Kieler Meeresforsch. 20, 18-29.

Kriss, A. E., 1961. Meeres-Mikrobiologie. A. d. Russ. Fischer, Jena, 570 pp.

LEDOYER, M., 1962. Étude de la faune vagile des herbiers superficiels de zosteracées et de quelques biotopes d'algues littorales. Recl. Traw. Stn. mar. Endonme 25 (39), 117-235.

MokievskiJ, O. B., 1960. Fauna litorali severo-zapadnogo poberežja Japonskogo morja. Trudy Inst. Okeanol. 34, 242-328.

Munda, I., 1962. Geographical and seasonal variations in the chemical composition of some Adriatic brown algae. Nova Hedwigia 4, 263-274.

- 1964. The quantity and chemical composition of Ascopbyllum nodosum (L.) LE JOL along the coast between the rivers Olfusá and Thjorsá (Southern Iceland). Botanica mar. 7, 77-88.

- 1967. Der Einfluß des Salzgehaltes auf die chemische Zusammensetzung, Wachstum und Fruktifizierung von einigen Fucaceen. Nova Hedwigia (im Druck).

Petrova, V., 1964. Fitoplanktont v Cerno more pred blgarskija brjag prez perioda 19581960 g. Izv. Inst, Ribov. Ribolov, Varna (Bull. Inst. Piscic. Pêche) 5, 5-32.

Reish, D. J., 1964. Studies on the Mytilus edulis community in Alamitos Bay, California. Veliger 6 (3), 124-131.

SEgerstRÅle, S. G., 1934. Studien über die Bodentierwelt in südfinnländischen Küstengewässern. Commentat. biol. 4 (8/9), 1-77.

- 1943. Weitere Studien über die Tierwelt der Fucus-Vegetation an der Südküste Finnlands. Commentat. biol. $9(4), 1-28$.

VAtova, A., 1949. La fauna bentonica dell'Alto e Medio Adriatico. Nova Tbalassia 1 (3), $1-110$.

Vinogradov, A. P., 1953. The elementary chemical composition of marine organisms. Sears Fdn mar. Res., New Haven, Conn., 647 pp. (Mem. No 2.)

ZavoDnk, D., 1963. Neka hidrografska opazanja u naseljima fitala okoline Rovinja. Hidrogr. Godisn. 1963, 189-193.

- 1965. Quelques résultats des recherches actuèlles sur les peuplements phytaux dans l'Adriatique du Nord. Rapp. P.-V. Réun. Commn int. Explor, scient. Mer Méditerr. 18, $101-106$.

Zenkević, L. A., 1956. Morja SSSR - ih fauna i flora. Akad. Nauk. SSSR, Moskva, 424 pp.

ZevinA, G. B., 1963. Obrastanie na Belom more. Trudy Inst. Okeanol. 70, 52-71. 